\title{
Cardiomyocyte D-dopachrome tautomerase protects against heart failure
}

\author{
Yina Ma, ${ }^{1,2}$ Kevin N. Su, ${ }^{1,3}$ Daniel Pfau, ${ }^{1,2}$ Veena S. Rao, ${ }^{1,2}$ Xiaohong Wu, ${ }^{1,2}$ Xiaoyue Hu, ${ }^{1,2}$ \\ Lin Leng, ${ }^{2}$ Xin Du, ${ }^{2}$ Marta Piecychna, ${ }^{2}$ Kenneth Bedi, ${ }^{4}$ Stuart G. Campbell, ${ }^{3,5}$ Anne Eichmann, ${ }^{1,2}$ \\ Jeffrey M. Testani, ${ }^{1,2}$ Kenneth B. Margulies, ${ }^{4}$ Richard Bucala, ${ }^{2}$ and Lawrence H. Young ${ }^{1,2,3}$ \\ ${ }^{1}$ Yale Cardiovascular Research Center, ${ }^{2}$ Department of Internal Medicine, and ${ }^{3}$ Department of Cellular \& Molecular \\ Physiology, Yale University School of Medicine, New Haven, Connecticut, USA. ${ }^{4}$ The Cardiovascular Research Institute, \\ University of Pennsylvania School of Medicine, Philadelphia, Pennsylvania, USA. ${ }^{5}$ Department of Biomedical Engineering, \\ Yale University School of Medicine, New Haven, Connecticut, USA.
}

\begin{abstract}
The mechanisms contributing to heart failure remain incompletely understood. o-dopachrome tautomerase (DDT) is a member of the macrophage migration inhibitory factor family of cytokines and is highly expressed in cardiomyocytes. This study examined the role of cardiomyocyte DDT in the setting of heart failure. Patients with advanced heart failure undergoing transplantation demonstrated decreased cardiac DDT expression. To understand the effect of loss of cardiac DDT in experimental heart failure, cardiomyocyte-specific DDT-KO (DDT-cKO) and littermate control mice underwent surgical transverse aortic constriction (TAC) to induce cardiac pressure overload. DDT-cKO mice developed more rapid cardiac contractile dysfunction, greater cardiac dilatation, and pulmonary edema after TAC. Cardiomyocytes from DDT-cKO mice after TAC had impaired contractility, calcium transients, and reduced expression of the sarcoplasmic reticulum calcium ATPase. The DDT-CKO hearts also exhibited diminished angiogenesis with reduced capillary density and lower VEGF-A expression after TAC. In pharmacological studies, recombinant DDT (rDDT) activated endothelial cell ERK1/2 and Akt signaling and had proangiogenic effects in vitro. The DDT-cKO hearts also demonstrated more interstitial fibrosis with enhanced collagen and connective tissue growth factor expression after TAC. In cardiac fibroblasts, rDDT had an antifibrotic action by inhibiting TCF- $\beta$-induced Smad-2 activation. Thus, endogenous cardiomyocyte DDT has pleiotropic actions that are protective against heart failure.
\end{abstract}

Conflict of interest: Yale University has been issued a patent (US 9,308,255 B2) for the use of D-dopachrome tautomerase in protection against ischemic injury. $\mathrm{RB}$ and $\mathrm{LHY}$ are coinventors and have received a patent licensing royalty from MIFCOR. RB has received founder's equity in MIFCOR. RB and LHY have received grant support from MIFCOR to study the use of recombinant DDT in ischemic tissue injury.

Copyright: (c) 2019, American Society for Clinical Investigation.

Submitted: April 1, 2019

Accepted: July 30, 2019

Published: September 5, 2019

Reference information: /CI Insight. 2019;4(17):e128900.

https://doi.org/10.1172/jci.

insight.128900.

\section{Introduction}

Heart failure is emerging as a major epidemiological problem and effective treatments are limited. The molecular mechanisms underlying adverse myocardial remodeling, pathological cardiac hypertrophy, and heart failure remain incompletely understood (1). Dysregulation of intracellular signaling cascades, including the Akt/mTOR $(2,3)$, calcineurin/nuclear factor of activated T cell (NFAT) (4), and MAPK pathways (5), have been implicated in altered metabolism, cell growth, calcium homeostasis, and myocyte contractility. The interactions between the cardiomyocyte, extracellular matrix, and vasculature, mediated by autocrine/paracrine factors, are also crucial to cardiac remodeling and the development of heart failure. In particular, cardiomyocyte-derived factors exert autocrine effects to regulate cardiomyocyte growth (6) and metabolism (7), while paracrine signals stimulate angiogenesis $(6,8-10)$ and regulate changes in the extracellular matrix (11).

The macrophage migration inhibitory factor (MIF) family cytokines are highly expressed in cardiomyocytes and exert autocrine and paracrine protective actions during cardiac ischemia/reperfusion ( 7 , 12). MIF is a pleiotropic cytokine that was first shown to have a pivotal role in the immune response (13) However, in cardiomyocytes, MIF expression is regulated by hypoxia-inducible factor 1 subunit $\alpha$ (14), and it is secreted during ischemia/reperfusion from preformed stores, activating the metabolic stress kinase, AMP-activated protein kinase (AMPK), which protects against ischemic injury (7).

D-dopachrome tautomerase (DDT or MIF-2) is a second member of the MIF family and shares $34 \%$ sequence identity with MIF (15-17). Although it was first detected in human melanoma and liver, DDT is now known to be ubiquitously expressed (18). Although it catalyzes the conversion of the nonphysio- 
logical enantiomer D-dopachrome to 5,6-dihydroxyindole, its physiological substrate(s) is unknown. DDT has been demonstrated to activate the MIF cell surface receptor CD74 (17), which transduces signals by interacting with CD44 (17). DDT exhibits a higher binding rate and a faster dissociation rate with CD74 when compared with MIF $(17,19)$. DDT also lacks the pseudo-ELR domain that allows MIF to interact as a noncognate ligand with the chemokine receptor CXCR2 (20). Through differential receptor binding, DDT lacks the negative inotropic effect of MIF to reduce cardiac contractility (21); consequently, DDT and MIF have distinct pharmacological actions in the heart (12).

Additional physiological actions of DDT have been elucidated in other tissues. For example, DDT secreted by adipocytes improves glucose intolerance observed in obesity (22). Similar to MIF, DDT also modulates cancer pathogenesis $(23,24)$. In endotoxemia and sepsis, the plasma concentration of DDT increases substantially and appears to have a detrimental proinflammatory action (17).

The regulation of DDT expression and its roles in cardiac hypertrophy and heart failure are not known. This study was designed to investigate the role of intrinsic cardiomyocyte-derived DDT in modulating the cardiac response to left ventricular (LV) pressure overload. Using a cardiomyocyte-specific DDT-KO mouse model, we assessed the effects of DDT deletion on cardiac remodeling and cell signaling and then investigated potential actions of DDT on endothelial cells and fibroblasts that might regulate the transition from hypertrophy to pathological heart failure. We also investigated the regulation of DDT expression in human heart failure, to test the hypothesis that loss of DDT may play a role in the progression to the advanced heart failure state.

\section{Results}

Downregulation of cardiac DDT expression in human heart failure. To test whether DDT or MIF expression is altered in advanced heart failure, myocardium from the left ventricle of explanted hearts from patients undergoing cardiac transplantation was analyzed by immunoblotting with specific antibodies. We included hearts from patients with ischemic and nonischemic cardiomyopathy. For comparison, myocardial tissue from nontransplanted donor hearts was assessed, including hearts that appeared normal and those that were found to have cardiac hypertrophy but normal contractility (Supplemental Table 1; supplemental material available online with this article; https://doi.org/10.1172/jci.insight.128900DS1).

Explanted failing human hearts had significantly reduced DDT protein and mRNA content compared with either normal or hypertrophied hearts (Figure 1). There was no difference evident in DDT expression between normal and hypertrophied nontransplanted hearts. MIF protein levels did not exhibit statistically significant differences between groups (Figure 1), although myocardial MIF mRNA content was lower in failing compared with hypertrophied hearts (not compared with controls). These results indicate differential downregulation of DDT and MIF and led to the hypothesis that a loss of myocardial DDT might contribute to the advanced stage of clinical human heart failure.

DDT and MIF expression in mouse heart after pressure overload. To assess the temporal regulation of DDT and MIF expression in the response to pressure overload, we performed immunoblots on LV homogenates from WT mice after transverse aortic constriction (TAC) or sham surgery. Results showed that cardiac DDT and MIF were both increased under pressure overload at 7 weeks after TAC, when LV function was still normal (Figure 2A), raising the possibility that they may have an early adaptive role in response to pressure overload. In contrast, at 12-17 weeks after TAC, when all mice had reduced LV contractile function (LV ejection fractions $<40 \%$ ), myocardial DDT protein content was reduced compared with sham-operated controls, but MIF protein content was normal (Figure 2B). These results indicate that DDT and MIF are differentially regulated in the late stages of heart failure in mice and are consistent with our observations in human heart failure.

Early cardiac contractile dysfunction and remodeling in cardiomyocyte-specific DDT-KO mice after pressure overload. To study the function of endogenous DDT in the cardiac response to LV pressure overload, cardiomyocyte-specific DDT-KO (DDT-cKO) male mice and littermate controls underwent TAC. DDTcKO mice were previously characterized (12) and show a highly efficient reduction of immunoreactive DDT protein, with minor residual $D d t$ mRNA and no compensatory upregulation of MIF (Supplemental Figure 1). There were no differences in the peak aortic velocity $(3.5 \pm 0.2 \mathrm{~m} / \mathrm{s}$ vs. $3.3 \pm 0.1 \mathrm{~m} / \mathrm{s}) \mathrm{mea}$ sured by Doppler 1 day after TAC in DDT-cKO versus control mice, indicating establishment of comparable aortic pressure gradients and LV pressure overload in the 2 groups. Cardiac structure and function were evaluated serially by echocardiography before and up to 6 weeks after surgery (Figure 3A). There 

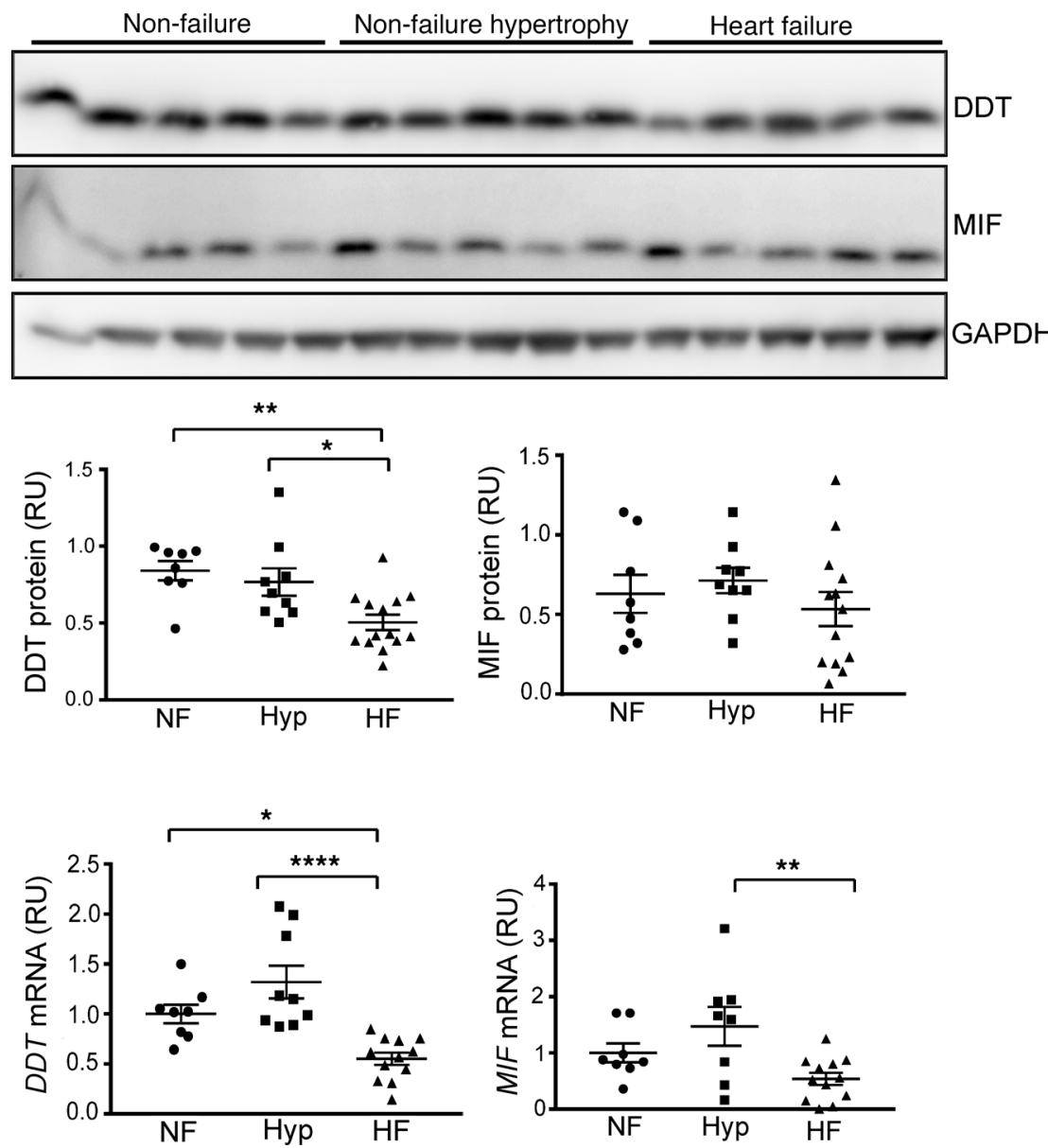

Figure 1. Cardiac DDT and MIF expression in human heart failure. LV myocardial tissue excised from nontransplanted donor nonfailing hearts (with or without hypertrophy) or from explanted hearts from patients undergoing cardiac transplantation for heart failure due to ischemic or nonischemic cardiomyopathy. Upper, immunoblots showing human heart tissue homogenate DDT, MIF, and GAPDH content in nonfailing, nonfailing hypertrophy, and cardiomyopathy hearts. Lower, human cardiac DDT and MIF protein and mRNA content quantified relative to GAPDH. NF, nonfailure; Hyp, nonfailure hypertrophy; $\mathrm{HF}$, heart failure; $\mathrm{RU}$, relative unit. Data are shown as mean $\pm \mathrm{SEM} ; n=8$ for the nonfailing heart and nonfailing hypertrophy heart; $n=12$ for the failing heart group. Significance determined by 1-way ANOVA with Tukey's multiple-comparisons test. ${ }^{*} P<0.05$; ${ }^{* *} P<0.01$; ${ }^{* * *} P<0.0001$ indicated by brackets.

were no differences in baseline LV size or contractile function between groups. However, compared with control mice, DDT-cKO mice developed rapid contractile dysfunction with deterioration in LV ejection fraction and cardiac output beginning at 2 weeks after TAC (Figure 3A and Supplemental Table 2). Both groups increased their LV wall thickness to a similar extent after TAC, but the DDT-cKO mice developed eccentric remodeling with LV dilatation and greater LV mass (Figure 3A). The left atrial size in DDTcKO mice was also more enlarged after TAC, consistent with greater atrial remodeling from heart failure (Figure 3A). In addition, DDT-cKO mice developed more pulmonary edema after TAC, as evidenced by an increased lung weight/body weight ratio, compared with control mice (Supplemental Table 3). The expression levels of mRNA transcripts coding for atrial natriuretic peptide and brain natriuretic peptide were greater in DDT-cKO versus control mice after TAC (Figure 3B). Taken together, these findings are indicative of more severe heart failure in the DDT-cKO mice.

To determine whether sex influences the role of DDT in modulating cardiac function during LV pressure overload, we performed separate experiments in DDT-cKO and control female mice. The DDT-cKO females also developed decreased LV ejection fractions at later time points and higher tissue content of natriuretic peptide mRNA transcripts, when compared with control female mice after TAC, although the functional differences appeared to be somewhat less pronounced compared with male mice (Supplemental Figure 2). 
A
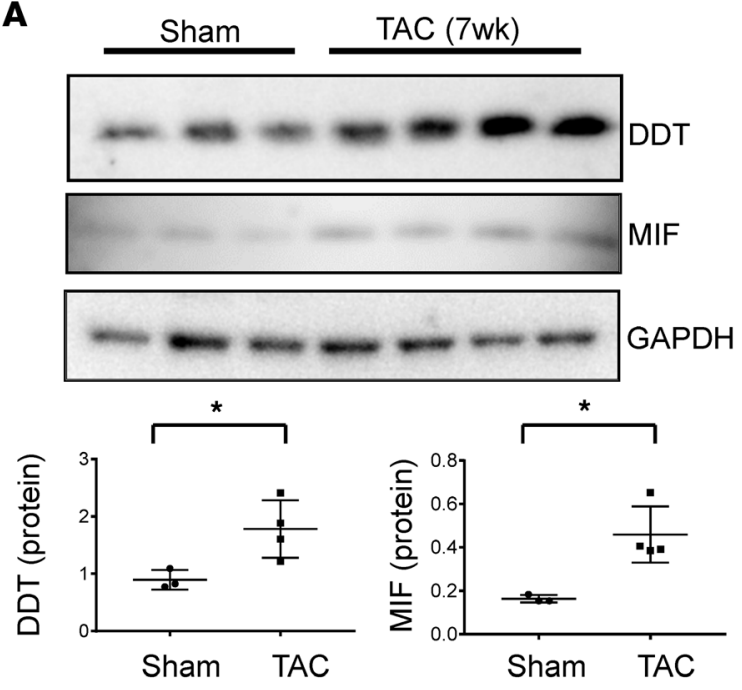

B
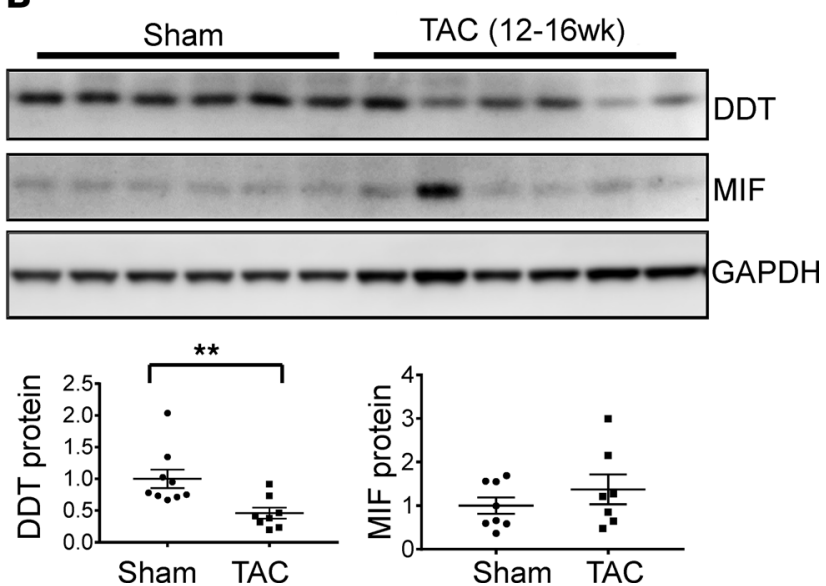

Figure 2. Cardiac DDT and MIF expression in WT mice after pressure overload. (A) Upper, immunoblots showing LV DDT, MIF, and GAPDH expression in mice 7 weeks after sham or transverse aortic constriction (TAC) surgery before the onset of heart failure. Lower, quantification of cardiac DDT and MIF protein contents relative to GAPDH. Data are shown as mean \pm SEM; $n=3$ mice for sham group; $n=4$ mice for TAC group. ${ }^{*} P<0.05$ compared with sham group. (B) Upper, immunoblots showing myocardial DDT, MIF, and GAPDH expression in mice 12-16 weeks after sham or TAC surgery following the onset of heart failure (all TAC mice had LV ejection fraction $<40 \%$ ). Lower, quantification of cardiac DDT and MIF protein contents relative to GAPDH. Data are shown as mean \pm SEM; $n=9$ mice for sham group; $n=8$ mice for TAC group. Significance determined by 2 -tailed Student's $t$ test. ${ }^{*} P<0.01$ compared with sham group.

Mice with global deletion of MIF have been reported to be more susceptible to heart failure after suprarenal abdominal aortic constriction (25). To directly compare the role of cardiomyocyte-derived DDT and MIF in the response to cardiac pressure overload, we generated an additional line of cardiomyocyte-specific MIF-KO (MIF-cKO) mice and subjected them to TAC. These mice had normal baseline cardiac size and contractile function. In contrast with DDT-cKO mice, the MIF-cKO mice demonstrated little susceptibility to heart failure, even when extending the observation period to 10 weeks after TAC (Supplemental Figure 3A). However, myocardial DDT expression was mildly increased and might have had a compensatory effect to prevent heart failure in the MIF-cKO mice (Supplemental Figure 3B). In contrast, we observed no compensatory increase in MIF expression in DDT-cKO mice after TAC (Supplemental Figure 4).

Differential cardiomyocyte remodeling in DDT-cKO mice after pressure overload. Individual cardiomyocyte cross-sectional area, measured on laminin-stained LV sections, was increased to a comparable extent in DDT-cKO and control mice after TAC (Figure 4A), consistent with the similar increase in LV wall thickness observed on echocardiograms (Figure 3A). Thus, the DDT-cKO mice did not appear to have exaggerated concentric LV hypertrophy. To further characterize cellular remodeling, isolated cardiomyocytes were obtained from digested hearts for width and length measurements. Cardiomyocyte width from control and DDT-cKO 

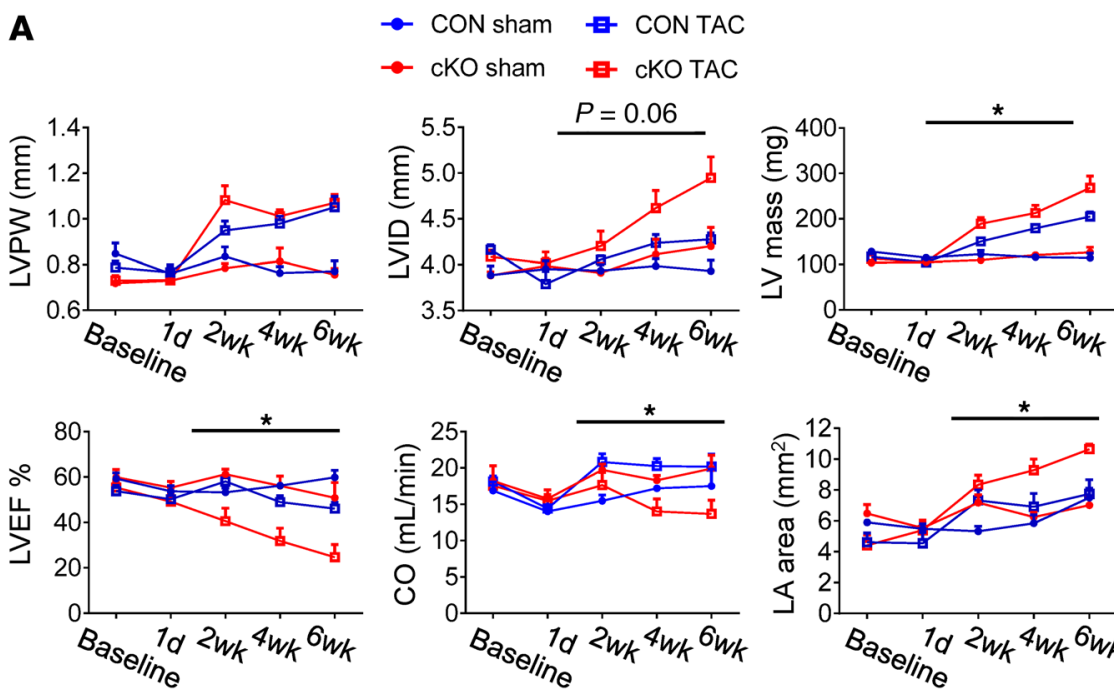

B 1 week after TAC
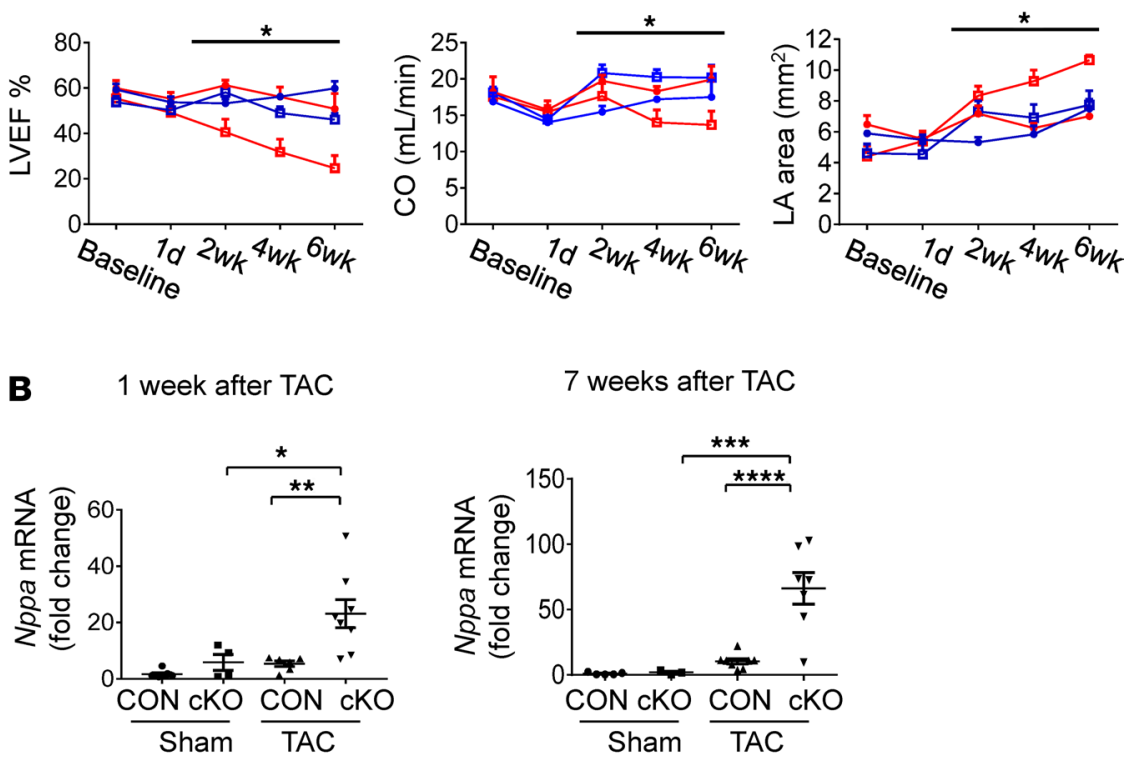

7 weeks after TAC
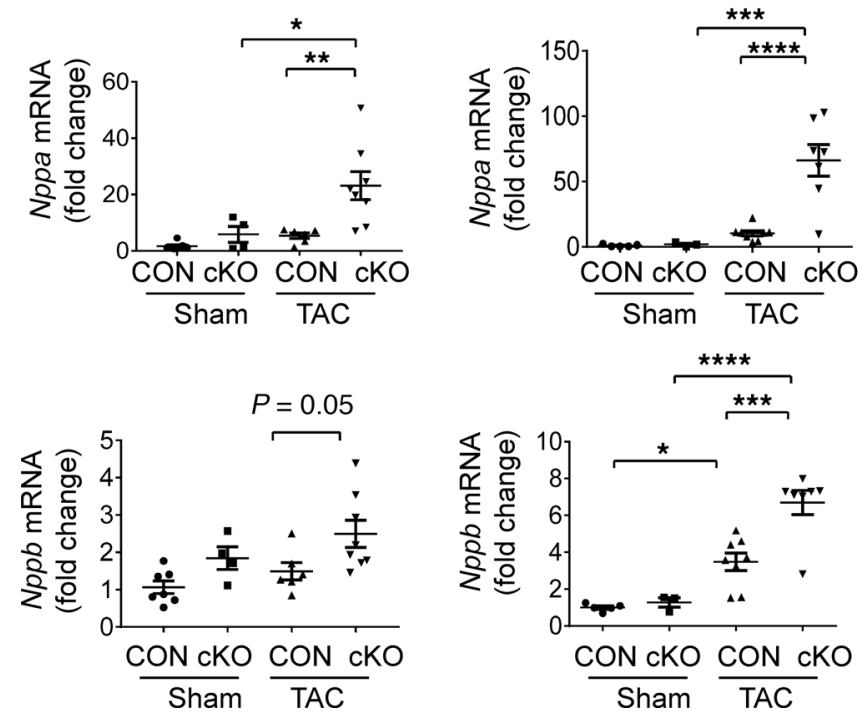

Figure 3. Effects of cardiomyocyte DDT deletion in mice on the transition from hypertrophy to heart failure after pressure overload. (A) Echocardiographic results in control (CON) mice and cardiomyocyte-specific DDT-KO (cKO) mice obtained 1 day before TAC or sham surgery and 1 day, 2 weeks, 4 weeks, and 6 weeks after surgery. LVPW, left ventricular posterior wall thickness; LVID, left ventricular inner diameter; LVEF, left ventricular ejection fraction; CO, cardiac output; LA, left atrium. Two-way ANOVA testing was used to analyze echocardiography results. ${ }^{*} P<0.05$ CON TAC vs. cKO TAC. (B) Heart failure biomarkers in CON and DDT-CKO mice after pressure overload; the content of mRNA transcripts for atrial natriuretic peptide ( $\mathrm{Nppa}$ ) and brain natriuretic peptide ( $\mathrm{Nppb}$ ) relative to ribosomal protein L32 (Rp/32) in the left ventricle 1 week and 7 weeks after sham or TAC surgery. Significance determined by 1-way ANOVA with Tukey's multiple-comparisons test. Data are shown as mean \pm SEM; $n=4-5$ mice for sham groups; $n=7-8$ mice for TAC groups. ${ }^{*} P<0.05 ;{ }^{* *} P<0.01 ;{ }^{* *} P<0.001 ;{ }^{* * *} P<0.0001$, indicated by brackets.

hearts was increased to a similar degree after TAC compared with their respective sham controls (Figure 4B), but the length of cardiomyocytes from DDT-cKO compared with control mice was slightly greater at 7 weeks after TAC (Figure 4B). The elongation of isolated cardiomyocytes together with the dilated LV dimensions on echocardiography indicate greater eccentric remodeling in DDT-cKO mice after TAC.

Impaired cardiomyocyte function and calcium handling in DDT-cKO mice after pressure overload. To determine whether the greater LV failure observed in DDT-cKO mice after TAC was due to an impairment in intrinsic cardiomyocyte contractility, cardiomyocytes were isolated 7 weeks after TAC (or sham) surgery to assess their contractile function and the calcium transients that are responsible for excitation-contraction coupling. 
A
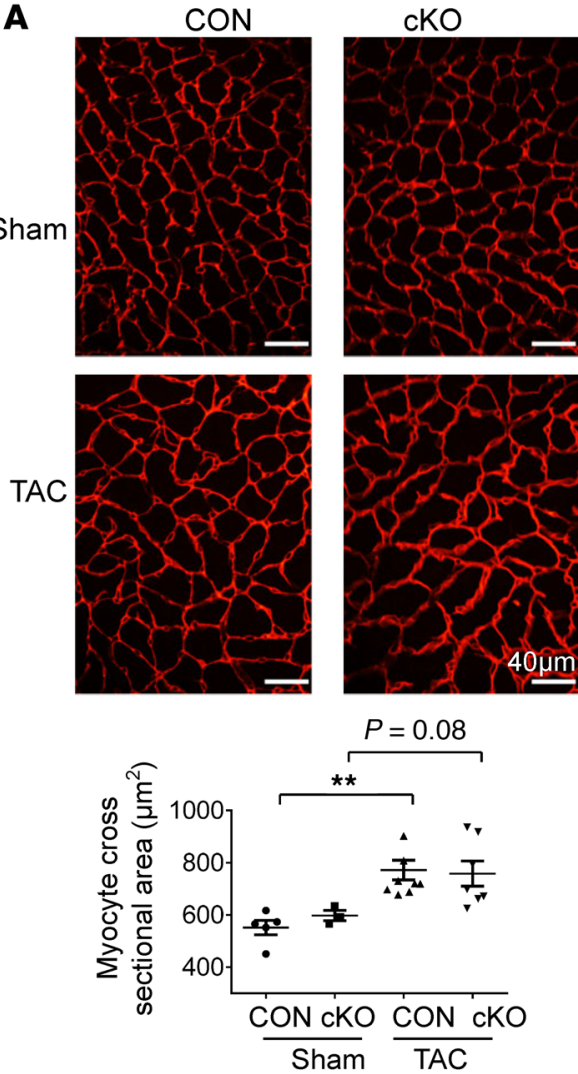

B
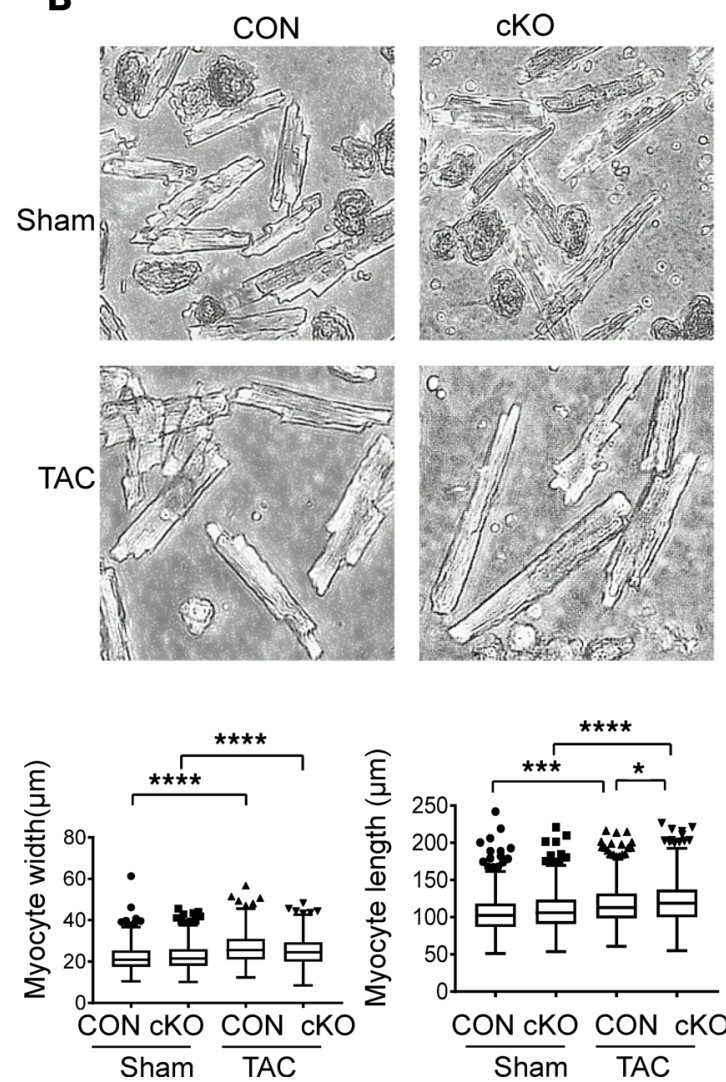

Figure 4. Effects of cardiomyocyte DDT deletion in mice on cardiomyocyte remodeling after pressure overload. (A) Cardiomyocyte perimeters were determined by laminin staining of heart LV sections. Cross-sectional area of cardiomyocytes was calculated from CON and cKO mice 7 weeks after sham or TAC surgery. $n=5-8$ mice per group, 4 images per mice, and 30-50 cells per image. (B) Cardiomyocyte width and length were determined in isolated cardiomyocytes from hearts 7 weeks after TAC surgery. All cells were imaged in the resting position with original magnification $\times 4$. Myocyte width and length were analyzed by Image). $n=200-300$ cardiomyocytes per group. Data are shown as mean \pm SEM. Significance determined by 1-way ANOVA with Tukey's multiple-comparisons test. ${ }^{*} P<0.05 ;{ }^{*} P<0.01$; ${ }^{* *} P<0.001$; ${ }^{* * *} P<0.0001$, indicated by brackets.

This analysis revealed a 37\% reduction in cardiomyocyte shortening in DDT-cKO versus control cardiomyocytes following TAC, indicating an intrinsic contractile deficit. The same cells showed a $36 \%$ decrease in both the magnitude of calcium transients and the calcium release velocity as well as a $30 \%$ decrease in the calcium uptake velocity of DDT-cKO compared with control cardiomyocytes after TAC (Figure 5A). To study the molecular mechanisms underlying the decreased calcium transients and contractility, we measured the expression of sarco-endoplasmic reticulum calcium ATPase (SERCA2a) and found that both SERCA2a protein and mRNA transcript levels were lower in DDT-cKO compared with control mice 1 week after TAC, when cardiac function was still maintained, and the early increase of wall thickness of DDT-cKO was comparable to controls (Supplemental Figure 5). Because Serca2a mRNA levels are negatively regulated

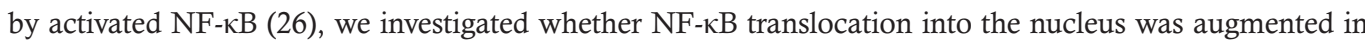
DDT-cKO hearts. Following subcellular fractionation, immunoblots showed an increase in NF- $\mathrm{kB} / \mathrm{p} 65$ and $\mathrm{NF}-\kappa \mathrm{B} / \mathrm{p} 50$ subunits in the nuclear fraction from DDT-cKO mice (Figure 5B), without evident change in the larger cytoplasmic fraction (Supplemental Figure 6). These results support the hypothesis that increased $\mathrm{NF}-\mathrm{\kappa B}$ activation might contribute to the suppression of SERCA2a expression and the associated defects in cardiomyocyte calcium handling and contractility in DDT-cKO mice after TAC.

Reduced angiogenesis in cKO mice after pressure overload. Angiogenesis parallels myocardial growth to maintain function during hypertrophic remodeling, and studies have shown that experimental models with impaired angiogenesis are prone to early heart failure during pressure overload (27). In hypertrophied hearts, angiogenesis results in an increase in the number of capillaries surrounding each cardiomyocyte (the capillary-to-myocyte ratio) (27), which is required to maintain perfusion and oxygen delivery to each of the hypertrophied cardiomy- 
A
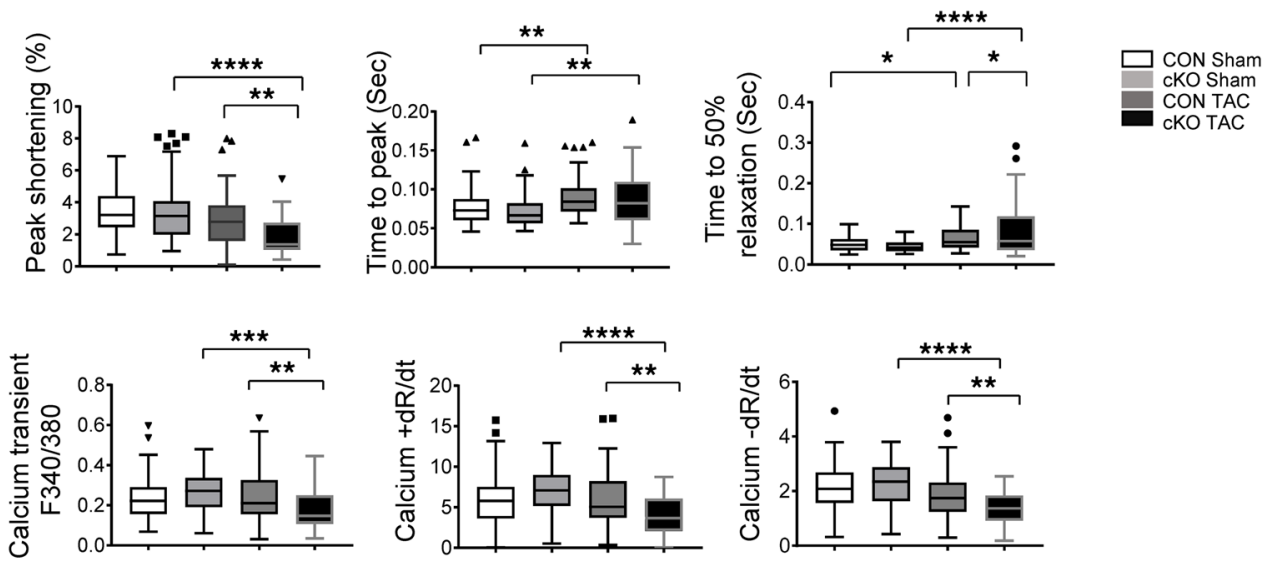

\section{B}
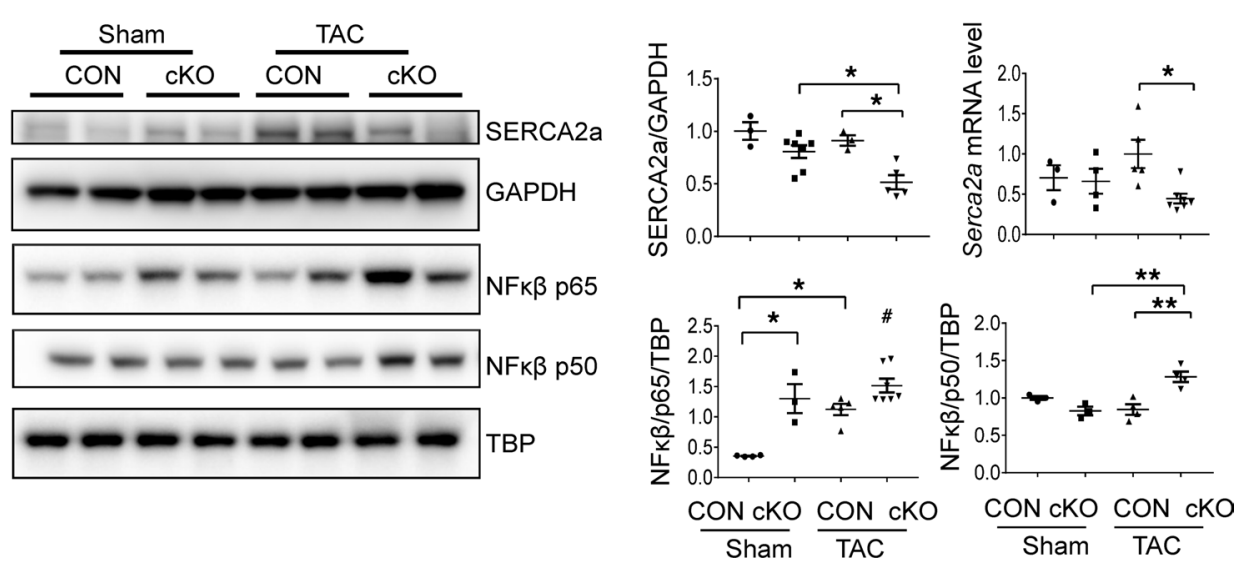

Figure 5. Effects of cardiomyocyte DDT deletion on cardiomyocyte contraction, calcium handling, and sarco-endoplasmic reticulum calcium ATPase expression after pressure overload. (A) Cellular contractile function and calcium handling in CKO and CON mice 7 weeks after sham or TAC surgery. Isolated cardiomyocytes were electrically stimulated at $1 \mathrm{~Hz}$. Sarcomere shortening, calcium transients, calcium uptake $(+\mathrm{dR} / \mathrm{dt})$, and release velocity $(-\mathrm{dR} / \mathrm{dt})$ were determined by Fura-2AM fluorescence in isolated cardiomyocytes. (B) Sarco-endoplasmic reticulum calcium ATPase (SERCA2a) expression in DDT-cKO mice 1 week after TAC. Immunoblots show SERCA2a content and NF- $\kappa B$ (p65 and p50) content in a nuclear (Nuc) extract of LV myocardium 1 week after TAC or sham surgery. CAPDH and TATA binding protein (TBP) were used as a cytoplasmic and a nuclear protein loading control, respectively. Data are shown as mean \pm SEM; $n=5-7$ mice per group. Significance determined by 1-way ANOVA with Tukey's multiple-comparisons test. ${ }^{*} P<$ $0.05 ;{ }^{* *} P<0.01 ;{ }^{* * *} P<0.0001$ indicated by brackets. ${ }^{*} P<0.05$ using 2 -tailed Student's $t$ test to compare the differences between the sham and TAC in the CKO and CON groups.

ocytes. We performed CD31 and laminin staining with confocal immunofluorescent imaging to assess capillaries and cardiomyocyte size, respectively. Histological analyses revealed that the adaptive increase in the capillary-to-myocyte ratio was smaller in DDT-cKO compared with control hearts 7 weeks after TAC (Figure 6A).

Given that VEGF-A is a major activator of angiogenesis in the heart (28), we assessed expression of VEGF-A to determine whether DDT might regulate it. We found a $40 \%$ decrease in VEGF-A protein on Western blotting of LV homogenates from the DDT-cKO compared with control mice 1 week after TAC (Figure 6B). Furthermore, Vegfa and Vegfb mRNA expression levels were also reduced in DDT-cKO compared with control mice (Figure 6B). These data together suggest that cardiomyocyte DDT regulates VEGF expression, which could contribute in part to defective angiogenesis observed in DDT-cKO hearts after TAC.

MIF is known to have direct proangiogenic properties in tumors (29), and we therefore hypothesized that cardiomyocyte-secreted DDT might have direct proangiogenic paracrine effects during LV pressure overload. Thus, we incubated HUVECs with human recombinant DDT (rDDT) and observed that $\mathrm{rDDT}$ induced a time-dependent activation of the proangiogenic ERK1/2 and Akt signaling path- 
A

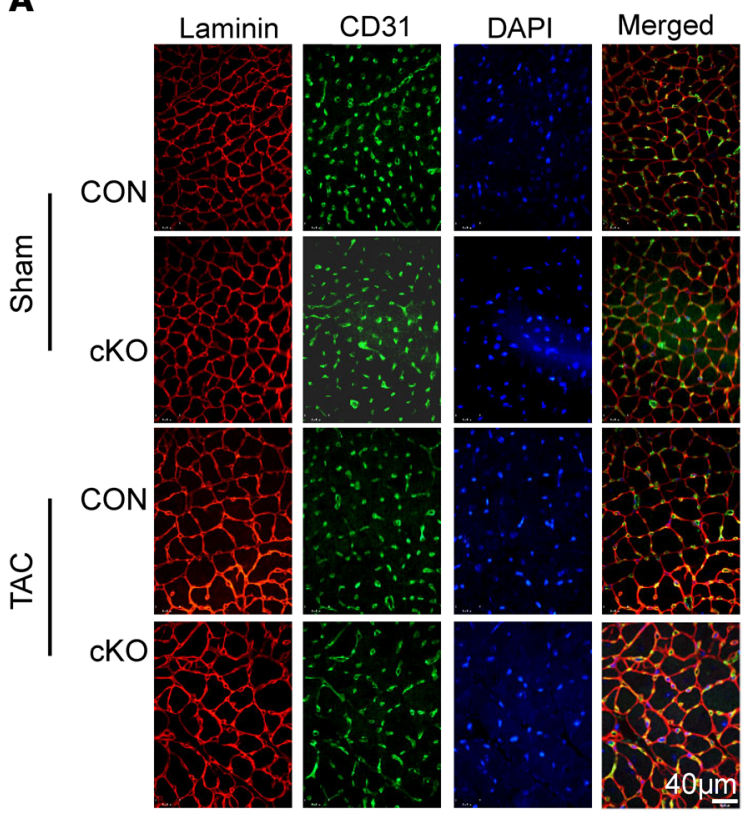

B
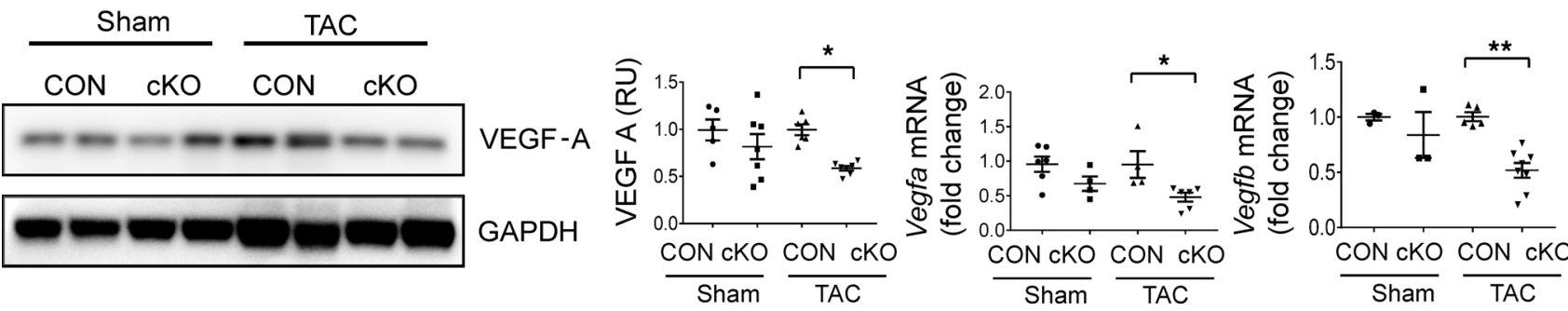

Figure 6. Effects of cardiomyocyte DDT deletion on cardiac angiogenesis and VEGF-A expression after pressure overload. (A) Immunofluorescence images stained for laminin (red), CD31 (green), and DAPI (blue) in LV sections of CON and cKO mice 7 weeks after sham or TAC surgery. Images were taken with confocal microscopy under original magnification $\times 30$. Right shows quantification of capillary and myocyte density and capillary-to-myocyte ratios. Data are shown as mean \pm SEM; $n=5-8$ mice per group. (B) VECF expression in CON and DDT-cKO mice 1 week after sham or TAC. Immunoblots show VEGF-A protein expression with quantification. Right shows Vegfa and Vegfb mRNA expression (quantitative PCR) in LV tissue homogenates. Significance determined by 1-way ANOVA with Tukey's multiple-comparisons test. ${ }^{*} P<0.05$; ${ }^{*} P<0.01$, indicated by brackets; ${ }^{*} P<0.05$ using 2 -tailed Student's $t$ test to compare the differences between the sham and TAC in the CKO and CON groups.

ways (Figure 7A). Compared with recombinant human VEGF (rhVEGF), rDDT treatment led to a comparable level of ERK1/2 activation but a lesser degree of Akt activation (Supplemental Figure 7). To further study the cellular actions of rDDT, endothelial cell cord formation was assessed in a Matrigel assay. rDDT treatment significantly increased cord formation and the number of nodes of endothelial cells after 20 hours of incubation (Figure 7B). These results indicate that rDDT has direct, activating effects on angiogenesis, presumably by stimulating endothelial Akt and ERK signaling, supporting the hypothesis that cardiomyocyte-derived DDT could have proangiogenic effects in the heart during pressure overload hypertrophy.

Increased fibrosis in DDT-cKO mice after pressure overload. Myocardial fibrosis leads to increased LV wall stiffness, which impairs myocardial relaxation and diastolic filling during the transition from hypertrophy to heart failure (30). To assess the degree of fibrosis after TAC, hearts were sectioned and stained with trichrome. Increased interstitial fibrosis was evident in DDT-cKO hearts as early as 1 week after TAC, in marked contrast with control TAC hearts that did not show any greater fibrosis compared with shams (Figure 8B). Although control mice subsequently developed fibrosis at 7 weeks after TAC, DDT-cKO mice continued to exhibit a significantly higher degree of fibrosis (Figure 8, A and C). Analysis of LV tissue was also performed to determine whether lack of cardiomyocyte DDT increased mRNA transcripts encoding extracellular matrix proteins and key growth factors that regulate matrix remodeling. Colla1 and Ctgf 
A
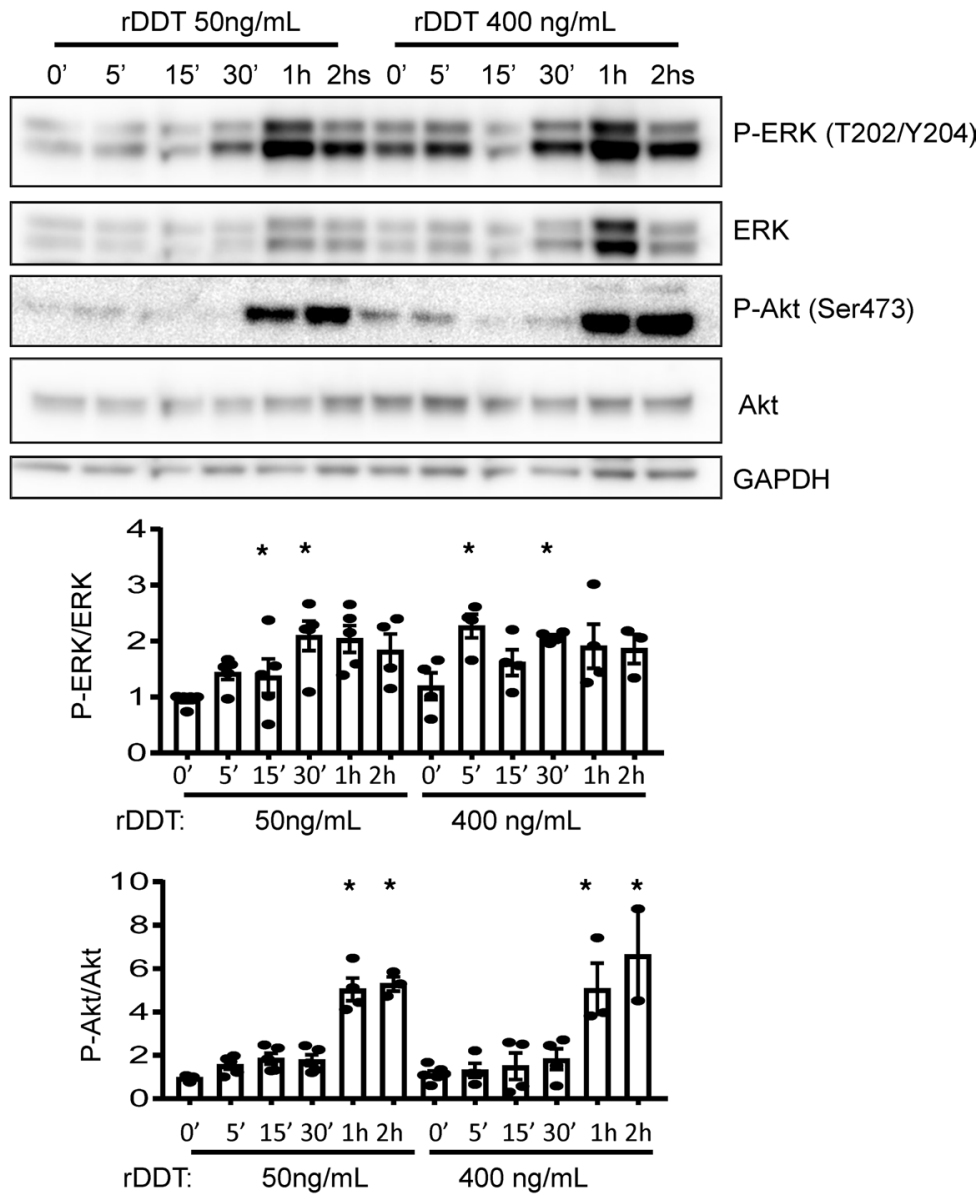

B

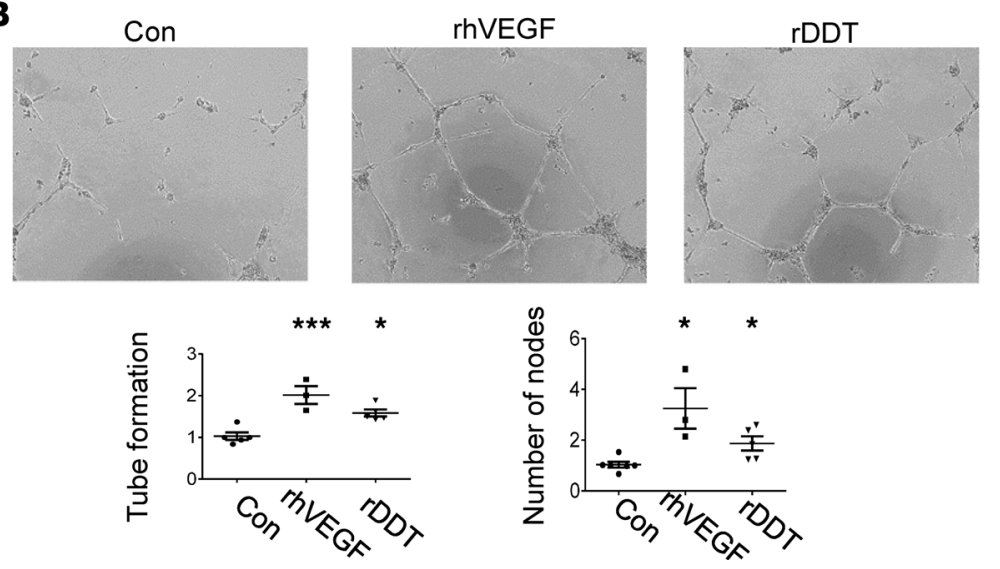

Figure 7. Effect of rDDT on the activation of proangiogenic signaling and the formation of endothelial cell cords and nodes. (A) Immunoblots with quantification showing Akt and ERK phosphorylation in HUVECs after treatment with rDDT at low $(50 \mathrm{ng} / \mathrm{mL})$ and high concentration $(400 \mathrm{ng} / \mathrm{mL})$. Significance determined by 1-way ANOVA with Tukey's multiple-comparisons test. ${ }^{*} P<0.05$ compared with its 0 -minute DDT treatment. (B) Upper, representative images of HUVECs after growth in Matrigel for 20 hours with rDDT $(400 \mathrm{ng} / \mathrm{mL})$, recombinant human VEGF (rhVEGF, $50 \mathrm{ng} / \mathrm{mL}$ ), or no treatment. Lower, quantification of number of tubes and nodes per image at original magnification $\times 4$. Data are shown as mean \pm SEM; $n=3-4$ independent studies. Significance determined by 1-way ANOVA with Tukey's multiple-comparisons test. ${ }^{*} P<$ $0.05 ;{ }^{* *} P<0.001$ vs. control.

mRNA (encoding collagen type I and connective tissue growth factor, respectively) levels were augmented at both 1 week and 7 weeks after TAC (Figure 8, B and C), while $M m p 3$ mRNA (encoding matrix metallopeptidase 3) levels were enhanced at 7 weeks after TAC in DDTcKO mice (Supplemental Figure 8).

MIF appears to have antifibrotic effects in the liver (31), and thus we hypothesized that cardiomyocyte-derived DDT might have direct antifibrotic effects. A cardinal event in the stimulation of fibroblasts is the activation of Smad- 2 by TGF- $\beta$, which stimulates extracellular matrix synthesis (32). We found that rDDT treatment diminished the phosphorylation of Smad-2 induced by TGF- $\beta$ in WT cardiac fibroblasts (Figure $8 D)$. To further test whether this action was mediated by the MIF receptor CD74, we isolated primary cardiac fibroblasts from WT and global CD74-KO mice. In the CD74-KO fibroblasts, DDT failed to suppress TGF- $\beta$ activation of Smad-2 (Figure 8E). These findings support the hypothesis that cardiomyocyte-derived DDT might have paracrine antifibrotic effects during LV pressure overload hypertrophy that are mediated by the fibroblast $\mathrm{CD} 74$ receptor.

Plasma DDT and MIF concentrations in patients with heart failure. Circulating plasma DDT concentrations are elevated during the normal newborn period, as well as in many disease conditions, including cancer, shock, and sepsis (17, 33). Plasma DDT concentrations differ considerably from local tissue content because they reflect the accumulation

of DDT released from both circulating inflammatory cells and many parenchymal tissues. To investigate whether circulating DDT concentrations are altered in an earlier stage of heart failure, we measured plasma DDT levels in a separate cohort of patients following intensive diuretic therapy in the hospital. For reference, we also measured plasma DDT concentrations in a group of volunteer blood donors at our clinical research center, who were younger, more often female, and less overweight (Supplemental Table 4). For comparison, we measured plasma MIF concentrations in these patients as well. The plasma concentrations of both DDT and MIF were increased in patients with heart failure compared with the reference group, independent of whether they had preserved or reduced LV ejection fractions (Figure 9A). Although there was no significant correlation between plasma DDT concentration and LV ejection fraction (Figure 9B), DDT was negatively correlated with plasma brain natriuretic peptide concentrations (Figure 9C). There was also a notable trend toward decreased survival in patients with lower plasma DDT concentrations (Figure 9D). In contrast, plasma MIF concentra- 
A

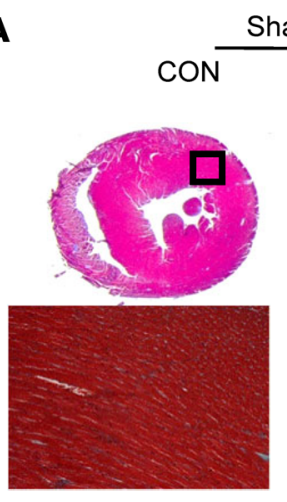

B

1 week after surgery
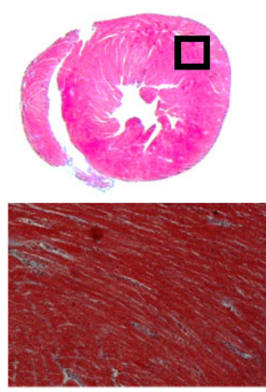

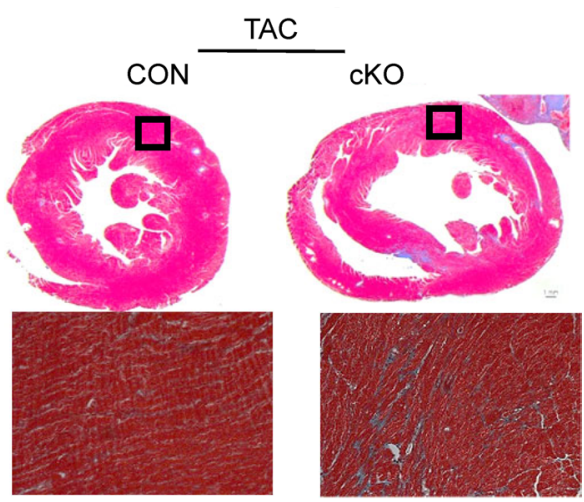

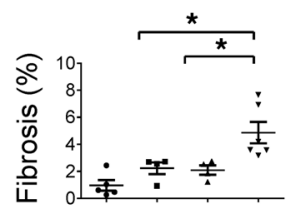
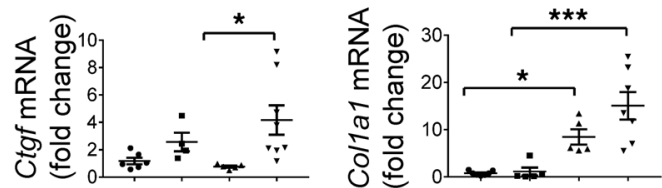

C

7 week after surgery
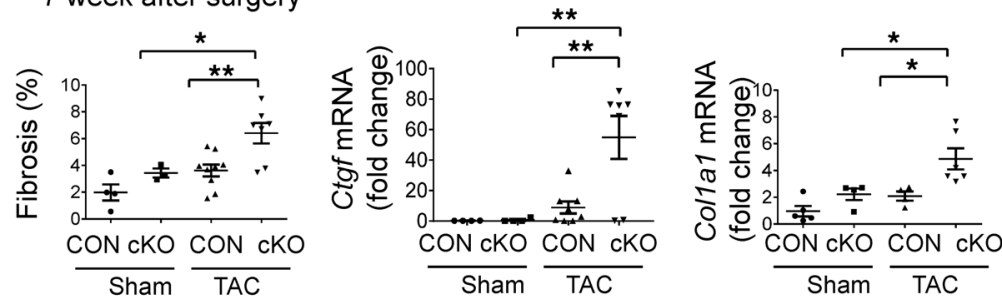

D
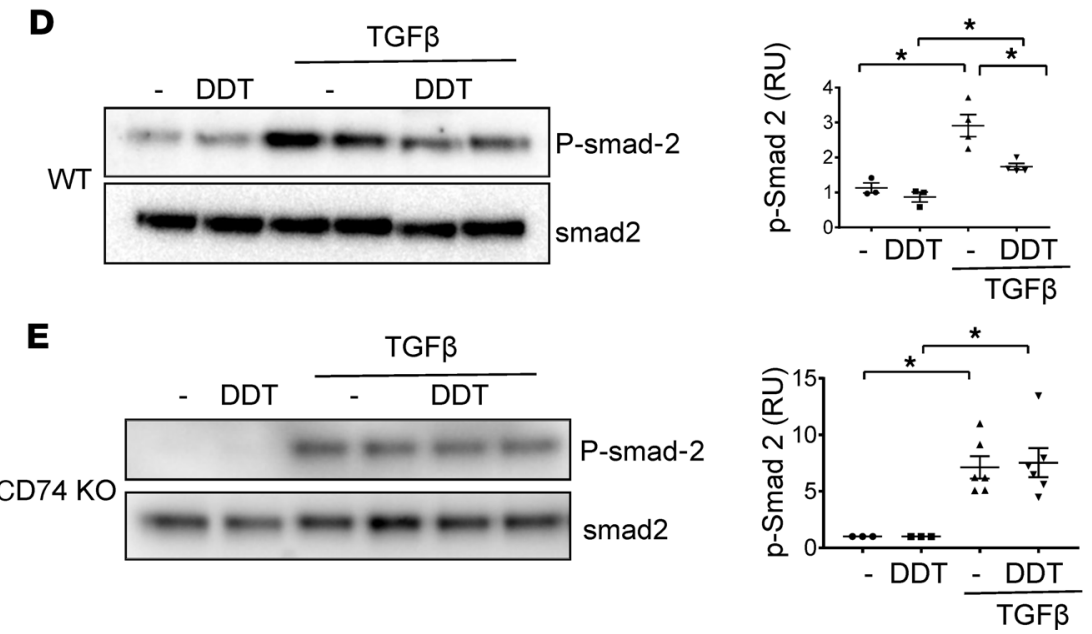

Figure 8. Effects of cardiomyocyte DDT deletion on myocardial fibrosis after pressure overload. (A) Representative trichrome-stained sections of the left ventricle in CON and CKO mice 7 weeks after TAC or sham surgery. Lower images are original magnification $\times 10$ of the myocardium in the area contained within the black box on the cross sections. (B and C) Connective tissue growth factor (Ctgf) and collagen type I $\alpha 1$ (Colla1) expression in DDT-cKO mice 1 and 7 weeks after TAC. Left graphs in $\mathbf{B}$ and $\mathbf{C}$ quantify the percentage of fibrosis at 1 and 7 weeks after surgery. Middle and right graphs quantify LV Ctgf and Col1a1 mRNA transcripts, respectively, at 1 and 7 weeks after surgery. These data are shown as mean \pm SEM; $n=4-7$ mice per group. (D) The effects of rDDT treatment on TCF- $\beta$-induced Smad- 2 phosphorylation in WT cardiac fibroblasts. Fibroblasts were isolated from WT mice hearts and incubated with or without TGF- $\beta(1 \mathrm{ng} / \mathrm{mL})$ for 1 hour and then treated with or without DDT $(400 \mathrm{ng} / \mathrm{mL})$ for 2 hours before cell lysis and immunoblotting. Graph shows the ratio of phosphorylated Smad-2 to total Smad-2. (E) The effects of rDDT treatment on TGF- $\beta$-induced Smad-2 phosphorylation in CD74-KO cardiac fibroblast. Fibroblasts were isolated from CD74-global KO mice hearts and incubated with or without TCF- $\beta(1 \mathrm{ng} / \mathrm{mL})$ for 1 hour and then treated with or without DDT $(400 \mathrm{ng} / \mathrm{mL})$ for 2 hours before cell lysis and immunoblotting. Graph shows the ratio of phosphorylated Smad-2 to total Smad-2. Data are shown as mean $\pm S E M ; n=3$ independent studies. Significance determined by 1-way ANOVA with Tukey's multiple-comparisons test. ${ }^{*} P<0.05$; ${ }^{* *} P<0.01$; ${ }^{* *} P<0.001$ indicated by brackets. 
tions did not significantly correlate with either plasma brain natriuretic peptide concentrations or LV ejection fraction (Figure 9, B and C), and there was no difference in survival between patients with lower and higher MIF plasma concentrations (Figure 9D).

\section{Discussion}

The results of this study demonstrate that cardiomyocyte-derived DDT has an important physiological role in heart failure. Cardiac DDT expression underwent initial adaptive upregulation and subsequent maladaptive downregulation after experimental pressure overload. Mice with cardiomyocyte-specific DDT deletion developed worse LV contractile dysfunction and dilatation with a much earlier onset of heart failure compared with controls. Heart failure was associated with impaired calcium handling (34), due in part to the downregulation of SERCA2a. In addition, cardiomyocyte DDT deletion adversely affected angiogenesis and interstitial fibrosis in cardiac hypertrophy. The proangiogenic effects of DDT may include upregulation of myocardial VEGF-A expression and possibly paracrine effects of secreted DDT to stimulate endothelial cell ERK and Akt signaling. The antifibrotic effects of DDT may be due to preventing increased myocardial Ctgf and Colla1 expression and possibly paracrine actions of secreted DDT to inhibit fibroblast Smad-2 activation by TGF- $\beta$. Finally, the results show that DDT expression is downregulated in failing hearts from patients with cardiomyopathy. Based on these observations, we postulate that loss of myocardial DDT may contribute to the development of advanced cardiomyopathy.

DDT and MIF have well-known actions as circulating proinflammatory cytokines at high concentrations (17). However, they are also highly expressed in the newborn period when they presumably modulate early postnatal growth (33). In addition, they are now recognized to have protective effects against injury in the heart (12), lung (35), liver (36), and kidney (37). We have shown that MIF and DDT have an intrinsic cardioprotective effect over the course of minutes during acute ischemia, through the rapid activation of AMPK (12). The current study elucidates a more long-term protective role of cardiomyocyte-derived DDT in heart failure, modulating not only autonomous cardiomyocyte contractile function but also the angiogenic and fibrotic responses that accompany pressure overload-induced cardiac hypertrophy. Thus, these results are potentially novel in describing the sustained, protective intraorgan pleiotropic actions of DDT to prevent maladaptive tissue remodeling.

The finding that DDT expression is downregulated in hearts explanted from patients undergoing heart transplant for advanced heart failure is of clinical interest. It is unlikely that DDT secretion and subsequent myocardial depletion account for the observed decrease in DDT content. First, DDT mRNA transcripts were also diminished. Second, although DDT is secreted from the ischemic heart (12), we observed similarly reduced cardiac DDT content in explanted hearts from patients with ischemic and nonischemic cardiomyopathies. We cannot exclude the possibility that clinical treatments used in patients with advanced heart failure might also have influenced cardiac DDT expression. Nonetheless, the results raise the possibility that acquired loss of DDT expression is a pathogenic mechanism in patients with advanced heart failure. Interestingly, there was less downregulation of MIF expression, indicating that these 2 MIF family members are differentially regulated and may have different roles in advanced human heart failure.

Plasma DDT also appeared mildly elevated in a separate group of patients with less advanced heart failure. The plasma DDT levels were not correlated with LV ejection fraction, but they were inversely related to brain natriuretic peptide. Patients with higher DDT plasma concentrations also tended to have a better prognosis after hospital discharge. These latter clinical results, although preliminary, lead to additional hypotheses that circulating DDT might have a protective myocardial effect and that plasma DDT might serve as a prognostic biomarker in patients with heart failure. Plasma concentrations and tissue levels of DDT appear to have directionally opposite changes in heart failure patients. These results suggest that there is differential regulation of DDT expression in the failing heart versus peripheral organs and blood cells. It is also noteworthy that the explanted hearts were perfused with cardioplegic solution to prevent contracture so that plasma DDT would not have confounded the measurement of myocardial DDT protein content.

Heart failure is typically accompanied by alterations in excitation-contraction coupling, with diminished calcium transients because of the blunted uptake and release of calcium from the sarcoplasmic reticulum (38). SERCA2a is the major pump responsible for calcium reuptake into the sarcoplasmic reticulum during diastole, and we identified a decrease in SERCA2a expression in DDT-cKO hearts early after pressure overload. This downregulation occurred before the onset of heart failure, in the 

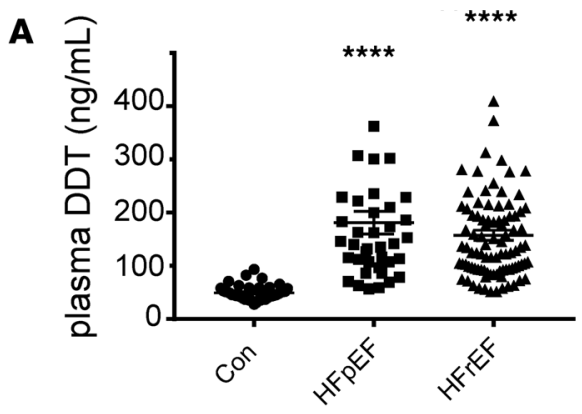

B

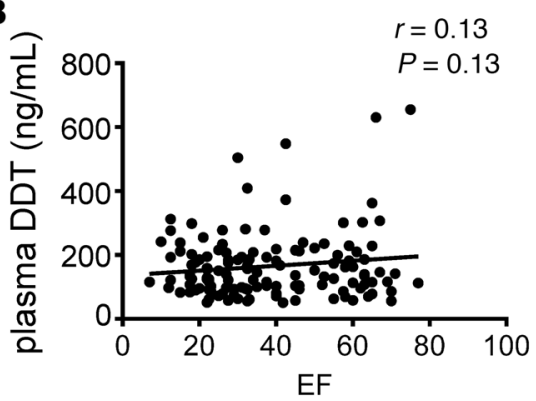

C

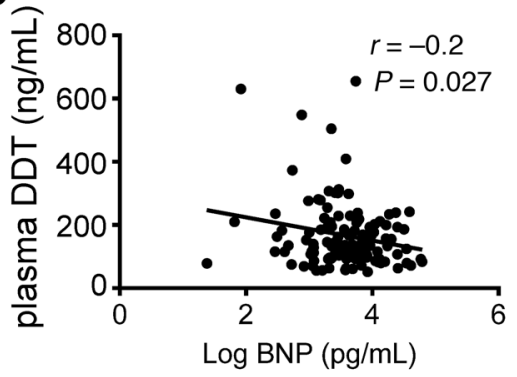

D

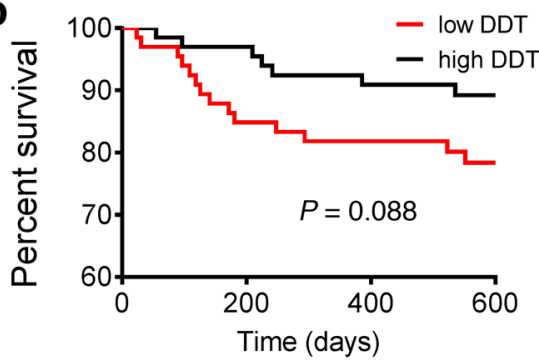

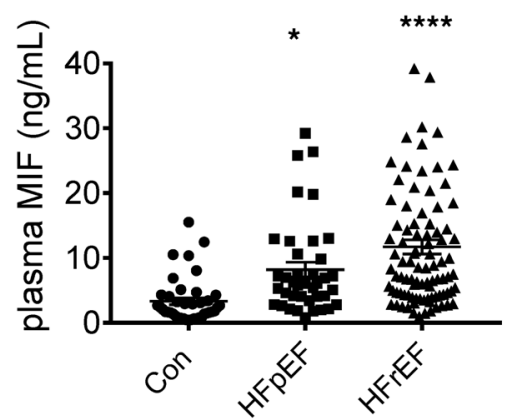
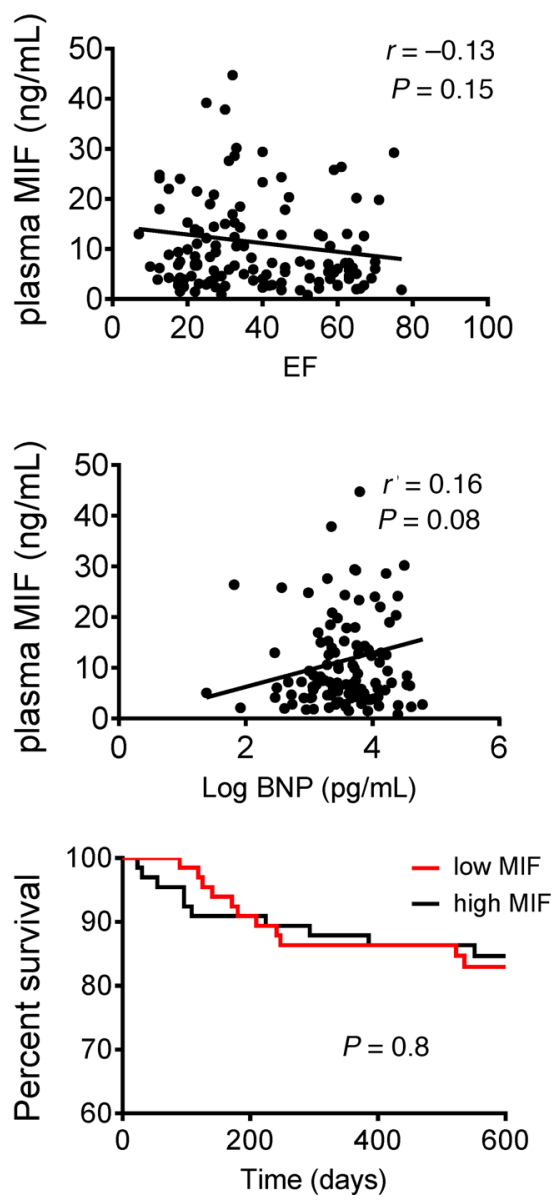

Figure 9. Plasma DDT and MIF concentrations in healthy individuals and patients with heart failure. (A) Plasma DDT and MIF concentrations were measured by specific ELISA in healthy controls (Con, $n=43$ ), heart failure with preserved ejection fraction (HFpEF, $n=41$ ), and heart failure with reduced ejection fraction (HFrEF, $n=99$ ). In the heart failure groups, plasma was measured following intensive diuretic therapy before discharge from the hospital. Control plasma was obtained from volunteer blood donors at our clinical research center. Data are shown as mean \pm SEM. Significance determined by 1-way ANOVA with Tukey's multiple-comparisons test. ${ }^{*} P<0.05 ;{ }^{* * *} P<0.0001$ compared with control. (B) Correlation between plasma DDT and MIF concentrations with LV ejection fraction (EF) in heart failure patients $(n=140)$. (C) Correlation between plasma DDT and MIF concentrations with log brain natriuretic peptide level in patients with heart failure $(n=140)$. Pearson's correlation was used for statistical comparison. (D) Kaplan-Meier survival curve comparing patients with heart failure $(n=140)$ with high and low DDT and MIF plasma concentrations. The median plasma concentrations of DDT $(135 \mathrm{ng} / \mathrm{mL})$ and MIF $(7.05 \mathrm{ng} / \mathrm{mL})$ were used to divide high versus low DDT and MIF groups. Log-rank test was used for statistical comparison.

absence of excessive LV hypertrophy. Thus, SERCA2a downregulation does not appear to be secondary to greater LV remodeling, but rather might have contributed to the diminished calcium transients and contractility that were measured in cardiomyocytes isolated from these hearts. The cKO hearts after pressure overload also showed increased translocation of $\mathrm{NF}-\kappa \mathrm{B}$ p50 and $\mathrm{NF}-\kappa \mathrm{B}$ p 65 into the nucleus, 
which has previously been reported to suppress SERCA2a transcription in a cardiomyocyte model (26). These results indicate that endogenous DDT inhibits NF- $\kappa \mathrm{B}$ activation during pressure overload but do not exclude a role for other mechanisms regulating SERCA2a, such as calcineurin/NFAT (39) and RhoA-ROCK-dependent serum response factor (40).

Angiogenesis is a hallmark feature of early hypertrophic growth, maintaining adequate blood flow to the enlarging cardiomyocytes. Impaired angiogenesis contributes to the transition from compensated hypertrophy to heart failure (27). We demonstrated a blunted increase in the capillary-to-myocyte ratio in DDT-cKO mice, suggesting that DDT is important in the adaptive angiogenic response to pressure overload. DDT may have both direct and indirect effects in promoting angiogenesis. Our results revealed diminished myocardial VEGF-A expression as early as 1 week after TAC in DDT-cKO mice, suggesting that loss of DDT might have an indirect effect to inhibit angiogenesis via downstream regulation of VEGF-A. DDT could regulate VEGF-A expression in cardiomyocytes or have paracrine effects on other cell types that secrete VEGF-A in the myocardium. rDDT had direct actions to increase endothelial cell tube formation and migration and activate the endothelial cell ERK and Akt signaling pathways that play a crucial role in angiogenesis (41). Akt suppresses apoptosis and stimulates migration of endothelial cells, while ERK modulates cell proliferation and migration during angiogenesis (28). Our findings are consistent with a prior report that DDT stimulates endothelial cell migration in a wound healing assay (23).

Interstitial fibrosis develops during the transition from compensated hypertrophy to pathological, decompensated heart failure (30). DDT-cKO hearts displayed early activation of profibrotic pathways, as evidenced by increased expression of Ctgf and Colla1, along with histological evidence of interstitial fibrosis as early as 1 week after pressure overload. Such early fibrosis was not observed in littermate controls and would be quite atypical in WT mice (42). Although DDT has not been previously shown to modulate fibrosis, there is a report that MIF has antifibrotic effects in the liver that are mediated through the CD74 receptor (31). In addition to blunting the increase in myocardial Ctgf during hypertrophy, we also observed that rDDT has a direct antifibrotic effect on isolated cardiac fibroblasts, which is also mediated by CD74. Treatment with $\mathrm{rDDT}$ inhibited TGF- $\beta$-induced phosphorylation Smad-2 (p-Smad-2) signaling, which is a key mechanism promoting interstitial fibrosis. Thus, we hypothesize that cardiomyocyte-derived DDT might have direct paracrine effects on cardiac fibroblasts during pressure overload.

These potentially novel findings extend our understanding of the diverse physiological functions of DDT in the heart. Prior work focused on the protective effect of cardiomyocyte-derived DDT released from preformed stores during acute cardiac ischemia (12). DDT activates the metabolic stress kinase AMPK via the CD74 receptor $(12,43)$. We previously showed that AMPK activation is impaired in DDT-cKO mice within minutes after the onset of cardiac ischemia (12). In contrast, the current study shows that cardiomyocyte-derived DDT has sustained pleiotropic effects in the heart that modulate pathological remodeling over the time course of weeks. Moreover, the action of DDT in pressure overload appears to have a distinct mechanism of action because we did not observe any difference in AMPK phosphorylation between control or DDT-cKO mice after TAC (data not shown).

In conclusion, cardiomyocyte DDT has pleiotropic effects that protect against pressure overloadinduced heart failure in a mouse model. The downregulation of DDT expression in advanced human heart failure leads to the hypothesis that loss of DDT might modulate disease progression. Further research is needed to further define the paracrine actions of DDT in the intact heart and the therapeutic potential of rDDT in heart failure.

\section{Methods}

Experimental animals. WT C57BL/6 male mice were obtained from the Charles River Laboratory. DDTcKO mice (Myh6-Cre Ddt $\left.t^{f / f f}\right)$ and littermate (Ddt $\left.{ }^{f / f f}\right)$ controls were generated as previously described (12). MIF-cKO mice (Myh6-Cre Mifflff) were generated by intercrossing Mifl/fl mice (44) with Myh6-Cre recombinase mice. Mif ${ }^{f / f l}$ mice were used as controls. Global CD74-KO mice were used for cardiac fibroblast isolation (12). All mice were on a pure C57BL/6 background.

TAC. TAC was performed between 9 and 12 weeks of age in mice using a minimally invasive approach, as previously described (45). After anesthesia with ketamine $(100 \mathrm{mg} / \mathrm{kg})$ and xylazine $(10 \mathrm{mg} / \mathrm{kg})$, a skin incision $0.5-1.0 \mathrm{~cm}$ in length was made at the level of the suprasternal notch, and a longitudinal cut 2-3 $\mathrm{mm}$ in length was made in the proximal portion of the sternum. This allowed for visualization of the aortic arch without opening the pleural space. A 27-gauge needle was placed next to the aortic arch to calibrate 
the constriction and an 8-0 suture was tied. Following ligation, the needle was removed. The skin was closed and mice were allowed to recover on a warming pad until they were fully awake. The sham procedure was identical except that the aorta was not constricted.

Echocardiography. Echocardiography was performed, as previously described (12). Mice were lightly anesthetized with $1 \%-2 \%(v / v)$ isoflurane and underwent 2-D and M-mode echocardiography (VisualSonics, Vevo 2100). Body temperature was maintained with a heating pad, and heart rate was monitored during the procedure. Echocardiography indexes of LV wall thickness and inner diameter were assessed with M-mode imaging from a short-axis view.

Matrigel assay. First, $30 \mu \mathrm{L}$ Matrigel (Corning, 354230) was dispersed in a 48-well plate and incubated for 30 minutes at $37^{\circ} \mathrm{C}$. Fifteen thousand HUVECs (Lonza) were plated in $300 \mu \mathrm{L}$ medium (EBM-2 [endothelial call basal medium-2] Lonzan with 5\% FBS), containing rhVEGF (R\&D Systems) (0-50 ng/mL) or human $\mathrm{rDDT}(0-400 \mathrm{ng} / \mathrm{mL}$, ref. 17). Imaging was conducted 16-20 hours after treatment, and the number of cords and nodes was quantified by ImageJ $(\mathrm{NIH})$.

Histological and immunofluorescence studies. Tissues were fixed with $4 \%(w / v)$ paraformaldehyde in PBS buffer overnight and transferred into $70 \%$ ethanol before paraffin wax embedding and sectioning. Masson's trichrome staining was performed by the Yale Histology Laboratory. Immunofluorescence was performed with antibodies against laminin (MilliporeSigma, L9393) and CD31 (R\&D Systems, AF3628).

Cardiomyocyte isolation and function measurement. First, 100 units of heparin was injected i.p. to prevent blood coagulation, and mice were then anesthetized with pentobarbital $(0.08 \mathrm{mg} / \mathrm{g}$ body weight). Hearts were excised and retrogradely perfused at $37^{\circ} \mathrm{C}$ with a Langendorff apparatus (ADInstruments) with a $\mathrm{Ca}^{2+}$-free Krebs-Henseleit buffer ( $\mathrm{pH} 7.3$ made in house) containing $0.6 \mathrm{mM} \mathrm{KH}_{2} \mathrm{PO}_{4}, 0.6 \mathrm{mM}$ $\mathrm{Na}_{2} \mathrm{HPO}_{4}, 10 \mathrm{mM}$ HEPES, $14.7 \mathrm{mM} \mathrm{KCl}, 1.7 \mathrm{mM} \mathrm{MgSO}_{4}, 120.3 \mathrm{mM} \mathrm{NaCl}, 4.6 \mathrm{mM} \mathrm{NaHCO}, 30$ $\mathrm{mM}$ taurine, $10 \mathrm{mM}$ glucose, and $15 \mathrm{mM}$ 2,3-butanedione monoxime that was bubbled with $95 \%$ $\mathrm{O}_{2} / 5 \% \mathrm{CO}_{2}$ (46). After stabilization, hearts were perfused with digestion buffer (perfusion buffer with $0.067 \mathrm{mg} / \mathrm{mL}$ Liberase Blendzyme 4, Roche) for 30 minutes. Upon completion of digestion, hearts were minced and filtered. Images were taken with an original magnification of $\times 4$, and cardiomyocyte size was calculated by ImageJ (NIH).

Cardiomyocyte mechanics and calcium handling studies. Cardiomyocyte mechanics and calcium handling studies were performed as previously described (47). After isolation, cardiomyocytes were incubated with progressively increasing calcium concentrations to reach a final calcium concentration of $1.8 \mathrm{mM}$. Cells were then resuspended in Tyrode solution (made from [in $\mathrm{mM}$ ] NaCl 140, $\mathrm{KCl} 5.4$, $\mathrm{CaCl} 2$ 1.8, $\mathrm{MgCl} 21$, Hepes 25, glucose 10) and loaded with $2.5 \mu \mathrm{mol} / \mathrm{L}$ Fura-2AM (Life Technologies) with pluronic acid $(2 \% \mathrm{w} / \mathrm{v})$ for 10 minutes. Cardiomyocyte calcium transients were measured using an inverted microscope (Nikon Eclipse) during constant perfusion with Tyrode solution at $37^{\circ} \mathrm{C}$. Cells were electrically stimulated at $1 \mathrm{~Hz}$, and contractile events were imaged in real time with a sarcomere-length camera system (HVSL, Aurora Scientific).

Primary cardiac fibroblast isolation. Cardiac primary fibroblasts were obtained from C57BL/6 or CD74-KO mice. Hearts were dissected in Hanks' balanced salt solution (MilliporeSigma) supplemented with an antibiotic and antimitotic solution (MilliporeSigma). Heart fragments were digested by a mix of $0.25 \%$ trypsin without EDTA (Gibco), collagenase IV (4 mg/mL, Worthington), and calcium chloride ( $0.3 \mathrm{mg} / \mathrm{mL}$, MilliporeSigma) for 1.5 hours at $37^{\circ} \mathrm{C}$. After mechanical dissociation, cells were passed through a cell strainer $(100 \mu \mathrm{m}$, Thermo Fisher Scientific). Cells were centrifuged at $435 \mathrm{~g}$ for 5 minutes, then resuspended in culturing medium (DMEM F-12 [Gibco by Life Technologies], 10\% FBS, penicillin/streptomycin, and 1\% L-glutamine). Cells were cultivated at $37^{\circ} \mathrm{C}$ in $5 \% \mathrm{CO}_{2}$. Medium was changed after 3 hours.

Western blotting. Western blots were performed as previously described (48). Heart lysates were resolved by SDS-PAGE and transferred onto polyvinylidene difluoride membranes (MilliporeSigma). Nuclear and cytoplasmic extraction were performed with a kit protocol (Thermo Fisher Scientific, 78833) The antibodies used were directed against MIF (17), DDT (17), VEGF (Santa Cruz Biotechnology, sc-152), GAPDH (Cell Signaling Technology, 2118s), p-Akt T308 (CST, 4056s), Akt (CST, 4691), p-ERK (CST, 4370s), ERK (CST, 9102c), p-Smad-2 (CST, 3101), Smad-2 (CST, 5339), SERCA2a (Thermo Fisher Scientific, MA3919) NF-кB p65 (CST, 8242), NF-кB p105/p50 (CST, 13586), and TBP (CST, 8515s).

Real-time PCR. Real-time PCR was carried out as previously described (49).

Human tissue research. Human LV myocardial samples for RNA and protein isolation were acquired from the University of Pennsylvania Human Heart Tissue Bank. Tissues from failing human hearts 
were obtained from patients receiving heart transplantation at the University of Pennsylvania. Nonfailing heart tissue was obtained from deceased organ donors without a history of heart failure through the Gift of Life donor program in Philadelphia. Prospective informed consent for research use of heart tissue was obtained from all transplant recipients and from the appropriate next of kin for the organ donors. All patient data and images were deidentified, and all protocols were performed in accordance with relevant guidelines for research involving tissue from human subjects. Tissue used in this study was collected and processed at the Cardiovascular Research Institute at the University of Pennsylvania between 2008 and 2017

Heart tissue procurement and processing. All human hearts received in situ high-potassium cardioplegia at the time of tissue procurement and were immediately placed on wet ice in $4^{\circ} \mathrm{C} \mathrm{Krebs-Henseleit} \mathrm{buffer.}$ After transport to the laboratory, within 4 hours of cardiectomy, the LV mass was determined on a scale after trimming off the right ventricular free wall, the atria, and the great vessels. Conventions for cardiac tissue sampling were as follows: LV free wall from a noninfarcted region, no septum, and predominantly anterior wall midway between the apex and base. Transmural tissues from the LV free wall were either snap-frozen in liquid nitrogen or fixed in $4 \%$ paraformaldehyde. Frozen tissue was placed in bar-coded cryovials and kept at $-80^{\circ} \mathrm{C}$ until use. Fixed tissues were washed in PBS and later processed, embedded in paraffin, sectioned, and stained. Immunoblot assays were performed by investigators blinded to the group assignment of the samples.

Blood sample collection. Patients receiving intravenous loop diuretics for the treatment of heart failure at Yale New Haven Hospital were considered for enrollment. Plasma samples used in this study were collected after initial decongestion and conversion from intravenous to oral diuretics.

ELISA. Human plasma MIF levels were measured using ELISA human MIF immunoassay according to the manufacturer's instructions (R\&D Systems hMIF ELISA kit). Plasma DDT levels of DDT were assessed with a DDT-specific sandwich ELISA as previously described (17).

Statistics. Data were expressed as mean \pm SEM. For single-factorial analyses, 2-tailed Student's $t$ test was performed to measure the differences in individual groups. One-way ANOVA with Tukey's multiple-comparisons test were used to determine the statistical significance between 2 or more independent groups. Two-way ANOVA with Tukey's multiple-comparisons test were used for echocardiography results analysis. Log-rank test was used for survival curve comparison. Correlation between 2 groups was assessed by Pearson's correlation coefficient. A $P$ value of less than 0.05 was considered statistically significant.

Comparisons between mice undergoing sham and TAC surgeries, in the DDT-cKO and control groups, were also performed in some analyses. Mean values were calculated for each group (DDT-cKO and control) and surgery type (TAC and sham). For each measure, a general linear regression model with group, type of surgery, and group-by-surgery-type terms was used to estimate the within-group effect of TAC (mean difference and 95\% confidence intervals) and to test for an interaction between surgery type and group. SAS v.9.4 was used for all analyses.

Study approval. All animal experiments were approved by the Yale Animal Care and Use Committee. All human study procedures were approved or waived by the Yale Human Investigation Committee or the University of Pennsylvania Institutional Review Board and by the Gift of Life Donor Program organ procurement organization.

\section{Author contributions}

YM, KNS, SGC, AE, JMT, KBM, RB, and LHY designed research studies. YM, KNS, DP, XW, XH, LL, $\mathrm{XD}, \mathrm{MP}$, and KB conducted experiments. YM, KNS, DP, VSR, XW, XH, LL, XD, MP, and KB acquired data. YM, KNS, DP, VSR, LL, XD, MP, SGC, AE, JMT, KM, RB, and LHY analyzed data. YM, KNS, DP, VSR, LL, AE, JMT, KBM, RB, and LHY wrote the manuscript.

\section{Acknowledgments}

The authors express their appreciation to Kimberly Clinton and Jeff Brandimarto, who assisted with subject consenting and tissue processing; the Gift of Life Donor Program of Philadelphia (Pennsylvania, USA), which facilitated human tissue procurement; and the patients and families who provided consent for research use of human heart tissues. This research was funded in part by a grant from the National Institutes of Health R01HL128069 to LHY and R01 AR049610 to RB. Procurement of human heart tissues was enabled by the National Institutes of Health HL089847 and HL105993 to KBM. 
Address correspondence to: Lawrence H. Young, Yale Cardiovascular Research Center, Department of Cardiovascular Medicine, Yale University School of Medicine, 300 George St., New Haven, Connecticut 06510, USA. Phone: 203.785.4102; Email: lawrence.young@yale.edu.

1. Maillet M, van Berlo JH, Molkentin JD. Molecular basis of physiological heart growth: fundamental concepts and new players. Nat Rev Mol Cell Biol. 2013;14(1):38-48.

2. Condorelli G, et al. Akt induces enhanced myocardial contractility and cell size in vivo in transgenic mice. Proc Natl Acad Sci US A. 2002;99(19):12333-12338.

3. Matsui T, et al. Phenotypic spectrum caused by transgenic overexpression of activated Akt in the heart. J Biol Chem. 2002;277(25):22896-22901.

4. Wilkins BJ, et al. Calcineurin/NFAT coupling participates in pathological, but not physiological, cardiac hypertrophy. Circ Res. 2004;94(1):110-118.

5. Kehat I, Molkentin JD. Extracellular signal-regulated kinase 1/2 (ERK1/2) signaling in cardiac hypertrophy. Ann N Y Acad Sci. 2010;1188:96-102.

6. van Wamel AJ, Ruwhof C, van der Valk-Kokshoom LE, Schrier PI, van der Laarse A. The role of angiotensin II, endothelin-1 and transforming growth factor-beta as autocrine/paracrine mediators of stretch-induced cardiomyocyte hypertrophy. Mol Cell Biochem. 2001;218(1-2):113-124.

7. Miller EJ, et al. Macrophage migration inhibitory factor stimulates AMP-activated protein kinase in the ischaemic heart. Nature. 2008;451(7178):578-582.

8. Giordano FJ, et al. A cardiac myocyte vascular endothelial growth factor paracrine pathway is required to maintain cardiac function. Proc Natl Acad Sci U S A. 2001;98(10):5780-5785.

9. Izumiya Y, Shiojima I, Sato K, Sawyer DB, Colucci WS, Walsh K. Vascular endothelial growth factor blockade promotes the transition from compensatory cardiac hypertrophy to failure in response to pressure overload. Hypertension. 2006;47(5):887-893.

10. Dzau VJ, Gibbons GH. Autocrine-paracrine mechanisms of vascular myocytes in systemic hypertension. Am J Cardiol. 1987;60(17):99I-103I.

11. Miura S, et al. Inhibition of matrix metalloproteinases prevents cardiac hypertrophy induced by beta-adrenergic stimulation in rats. J Cardiovasc Pharmacol. 2003;42(2):174-181.

12. Qi D, et al. The vestigial enzyme D-dopachrome tautomerase protects the heart against ischemic injury. J Clin Invest. 2014;124(8):3540-3550.

13. Calandra T, Roger T. Macrophage migration inhibitory factor: a regulator of innate immunity. Nat Rev Immunol. 2003;3(10):791-800

14. Baugh JA, Gantier M, Li L, Byrne A, Buckley A, Donnelly SC. Dual regulation of macrophage migration inhibitory factor (MIF) expression in hypoxia by CREB and HIF-1. Biochem Biophys Res Commun. 2006;347(4):895-903.

15. Odh G, Hindemith A, Rosengren AM, Rosengren E, Rorsman H. Isolation of a new tautomerase monitored by the conversion of D-dopachrome to 5,6-dihydroxyindole. Biochem Biophys Res Commun. 1993;197(2):619-624.

16. Sugimoto H, Taniguchi M, Nakagawa A, Tanaka I, Suzuki M, Nishihira J. Crystal structure of human D-dopachrome tautomerase, a homologue of macrophage migration inhibitory factor, at 1.54 A resolution. Biochemistry. 1999;38(11):3268-3279.

17. Merk M, et al. The D-dopachrome tautomerase (DDT) gene product is a cytokine and functional homolog of macrophage migration inhibitory factor (MIF). Proc Natl Acad Sci U S A. 2011;108(34):E577-E585.

18. Esumi N, Budarf M, Ciccarelli L, Sellinger B, Kozak CA, Wistow G. Conserved gene structure and genomic linkage for D-dopachrome tautomerase (DDT) and MIF. Mamm Genome. 1998;9(9):753-757.

19. Leng L, et al. MIF signal transduction initiated by binding to CD74. J Exp Med. 2003;197(11):1467-1476.

20. Bernhagen J, et al. MIF is a noncognate ligand of CXC chemokine receptors in inflammatory and atherogenic cell recruitment. Nat Med. 2007;13(5):587-596.

21. Weber C, et al. Structural determinants of MIF functions in CXCR2-mediated inflammatory and atherogenic leukocyte recruitment. Proc Natl Acad Sci U S A. 2008;105(42):16278-16283.

22. Iwata $\mathrm{T}$, et al. The action of D-dopachrome tautomerase as an adipokine in adipocyte lipid metabolism. PLoS ONE. 2012;7(3):e33402.

23. Pasupuleti V, et al. Dysregulated D-dopachrome tautomerase, a hypoxia-inducible factor-dependent gene, cooperates with macrophage migration inhibitory factor in renal tumorigenesis. J Biol Chem. 2014;289(6):3713-3723.

24. Hussain F, et al. Human anti-macrophage migration inhibitory factor antibodies inhibit growth of human prostate cancer cells in vitro and in vivo. Mol Cancer Ther. 2013;12(7):1223-1234.

25. Xu X, Hua Y, Nair S, Bucala R, Ren J. Macrophage migration inhibitory factor deletion exacerbates pressure overload-induced cardiac hypertrophy through mitigating autophagy. Hypertension. 2014;63(3):490-499.

26. Huang H, Joseph LC, Gurin MI, Thorp EB, Morrow JP. Extracellular signal-regulated kinase activation during cardiac hypertrophy reduces sarcoplasmic/endoplasmic reticulum calcium ATPase 2 (SERCA2) transcription. J Mol Cell Cardiol. 2014;75:58-63

27. Shiojima I, et al. Disruption of coordinated cardiac hypertrophy and angiogenesis contributes to the transition to heart failure. $J$ Clin Invest. 2005;115(8):2108-2118.

28. Simons M, Gordon E, Claesson-Welsh L. Mechanisms and regulation of endothelial VEGF receptor signalling. Nat Rev Mol Cell Biol. 2016;17(10):611-625.

29. Chesney J, Metz C, Bacher M, Peng T, Meinhardt A, Bucala R. An essential role for macrophage migration inhibitory factor (MIF) in angiogenesis and the growth of a murine lymphoma. Mol Med. 1999;5(3):181-191.

30. Conrad CH, Brooks WW, Hayes JA, Sen S, Robinson KG, Bing OH. Myocardial fibrosis and stiffness with hypertrophy and heart failure in the spontaneously hypertensive rat. Circulation. 1995;91(1):161-170. 
31. Heinrichs D, et al. Macrophage migration inhibitory factor (MIF) exerts antifibrotic effects in experimental liver fibrosis via CD74. Proc Natl Acad Sci U S A. 2011;108(42):17444-17449.

32. Meng XM, Nikolic-Paterson DJ, Lan HY. TGF- $\beta$ : the master regulator of fibrosis. Nat Rev Nephrol. 2016;12(6):325-338.

33. Roger $\mathrm{T}$, et al. Plasma levels of macrophage migration inhibitory factor and d-dopachrome tautomerase show a highly specific profile in early life. Front Immunol. 2017;8:26.

34. Balke CW, Shorofsky SR. Alterations in calcium handling in cardiac hypertrophy and heart failure. Cardiovasc Res. 1998;37(2):290-299

35. Coleman AM, et al. Cooperative regulation of non-small cell lung carcinoma angiogenic potential by macrophage migration inhibitory factor and its homolog, D-dopachrome tautomerase. J Immunol. 2008;181(4):2330-2337.

36. Hiyoshi M, et al. D-Dopachrome tautomerase is a candidate for key proteins to protect the rat liver damaged by carbon tetrachloride. Toxicology. 2009;255(1-2):6-14.

37. Stoppe $\mathrm{C}$, et al. Interaction of MIF family proteins in myocardial ischemia/reperfusion damage and their influence on clinical outcome of cardiac surgery patients. Antioxid Redox Signal. 2015;23(11):865-879.

38. Periasamy M, Huke S. SERCA pump level is a critical determinant of $\mathrm{Ca}(2+)$ homeostasis and cardiac contractility. $J$ Mol Cell Cardiol. 2001;33(6):1053-1063.

39. Anwar A, Schlüter KD, Heger J, Piper HM, Euler G. Enhanced SERCA2A expression improves contractile performance of ventricular cardiomyocytes of rat under adrenergic stimulation. Pflugers Arch. 2008;457(2):485-491.

40. Vlasblom R, et al. RhoA-ROCK signaling is involved in contraction-mediated inhibition of SERCA2a expression in cardiomyocytes. Pflugers Arch. 2009;458(4):785-793.

41. Magrane J, Christensen RA, Rosen KM, Veereshwarayya V, Querfurth HW. Dissociation of ERK and Akt signaling in endothelial cell angiogenic responses to beta-amyloid. Exp Cell Res. 2006;312(7):996-1010.

42. Huusko J, et al. AAV9-mediated VEGF-B gene transfer improves systolic function in progressive left ventricular hypertrophy. Mol Ther. 2012;20(12):2212-2221.

43. Ma Y, Li J. Metabolic shifts during aging and pathology. Compr Physiol. 2015;5(2):667-686.

44. Pellowe AS, et al. Endothelial cell-secreted MIF reduces pericyte contractility and enhances neutrophil extravasation. FASEB J. 2019;33(2):2171-2186

45. Li N, et al. miR-182 modulates myocardial hypertrophic response induced by angiogenesis in heart. Sci Rep. 2016;6:21228.

46. Chen S, et al. Alpha1 catalytic subunit of AMPK modulates contractile function of cardiomyocytes through phosphorylation of troponin I. Life Sci. 2014;98(2):75-82.

47. Kuo IY, Kwaczala AT, Nguyen L, Russell KS, Campbell SG, Ehrlich BE. Decreased polycystin 2 expression alters calcium-contraction coupling and changes $\beta$-adrenergic signaling pathways. Proc Natl Acad Sci U S A. 2014;111(46):16604-16609.

48. Ma Y, et al. Antithrombin up-regulates AMP-activated protein kinase signalling during myocardial ischaemia/reperfusion injury. Thromb Haemost. 2015;113(2):338-349.

49. Kim GE, et al. LKB1 deletion causes early changes in atrial channel expression and electrophysiology prior to atrial fibrillation. Cardiovasc Res. 2015;108(1):197-208. 